\title{
Novel Prostate Specific Antigen plastic antibody designed with charged binding sites for an improved protein binding and its application in a biosensor of potentiometric transduction
}

\author{
Tânia S.C.R. Rebelo, C. Santos, J. Costa-Rodrigues, M.H. Fernandes, \\ João P. Noronha, M. Goreti F. Sales
}

\begin{abstract}
This work shows that the synthesis of protein plastic antibodies tailored with selected charged monomers around the binding site enhances protein binding. These charged receptor sites are placed over a neutral polymeric matrix, thus inducing a suitable orientation the protein reception to its site. This is confirmed bypreparing control materials with neutral monomers and also with non-imprinted template. This concept has been applied here to Prostate Specific Antigen (PSA), the protein of choice for screening prostate can-cer throughout the population, with serum levels $>10 \mathrm{ng} / \mathrm{mL}$ pointing out a high probability of associated cancer.

Protein Imprinted Materials with charged binding sites (C/PIM) have been produced by surface imprinting over graphene layers to which the protein was first covalently attached. Vinylben- zyl(trimethylammonium chloride) and vinyl benzoate were introduced as charged monomers labelling the binding site and were allowed to self-organize around the protein. The subsequent polymerization was made by radical polymerization of vinylbenzene. Neutral PIM (N/PIM) prepared without oriented charges and non imprinted materials (NIM) obtained without template were used as controls.

These materials were used to develop simple and inexpensive potentiometric sensor for PSA. They were included as ionophores in plasticized PVC membranes, and tested over electrodes of solid or liq- uid conductive contacts, made of conductive carbon over a syringe or of inner reference solution over micropipette tips. The electrodes with charged monomers showed a more stable and sensitive response,

with an average slope of $-44.2 \mathrm{mV} /$ decade and a detection limit of $5.8 \times 10^{-11} \mathrm{~mol} / \mathrm{L}(2 \mathrm{ng} / \mathrm{mL})$. The cor-

responding non-imprinted sensors showed lower sensitivity, with average slopes of $-24.8 \mathrm{mV} /$ decade. The best sensors were successfully applied to the analysis of serum, with recoveries ranging from 96.9 to $106.1 \%$ and relative errors of $6.8 \%$.
\end{abstract}

Keywords:

Prostate Specific Antigen, Potentiometry Graphene layers, Ion selective sensors, Molecularly-imprinted polymers, Serum.

\section{Introduction}

According to recent data from World Health Organisation (WHO), prostate cancer is the commonest form of cancer in men in Europe [1]. Accurate and early detection of prostate cancer is very important in this context, attributing early diagnosis a major role in the successful treatment of the disease.
A successful early diagnosis means that early alterations in the body should belooked for. A non-invasive approach is important in this context, allowing its application throughout the male population. So far, the only medical recommendation for prostate cancer early screening suggests monitoring the levels of Prostate Specific Antigen (PSA) in serum, in men over 45 years old. Monitoring PSA levels to follow up the evolution of prostate cancer disease is also recommended, especially for being a non-invasive procedure.

PSA is a glycoprotein with a molecular mass of approximately $33 \mathrm{kDa}$, produced by the secretory epithelium of human prostate [2]. A total PSA level in the blood $<4 \mathrm{ng} / \mathrm{mL}$ indicates that prostate 

cancer is improbable, while PSA levels $>10 \mathrm{ng} / \mathrm{mL}$ mean cancer is likely [3]; values ranging from $4-10 \mathrm{ng} / \mathrm{mL}$ are in a gray zone. Currently, the standard methods available for PSA screening are immunoassays, like Enzyme-Linked Immunosorbent Assay (ELISA). These methods are highly sensitive and specific for the detection of PSA, but are also very expensive because they require specific natural antibodies, with special handling and storage conditions. In addition, there is a great affinity between the antibody and its antigen, being impossible to dissociate these two biological components after binding. This feature turns the method of irreversible nature and of single use.

Biosensors have emerged in recent years as an attractive tool to carry out quick and local clinical analysis [4], being today an alternative concept to some ELISA methods. Several biosensors for PSA have also been reported, with detection limits ranging from 0.1 to $8 \mathrm{ng} / \mathrm{mL}$. Although they use different transduction modes, varying from electrochemical [3,5-8], optical [9-13], frequency [14] to mass [15-17] detection methods, the recognition element is always an antibody. The selection of an antibody as biological receptor confers the biosensor a selective response for PSA, but the drawbacks mentioned before for the ELISA methods also apply to these.

As an alternative, a new PSA biosensor could use artificial antibodies instead of the natural ones. These are synthetic materials offering higher chemical/thermal stability than their natural counterparts [4], and that promote a reversible response, thus enabling an 'infinite' reuse of the material. Protein plastic antibodies are PIM typically obtained by surface imprinting procedures [18], where the polymeric matrix is grown around the protein and the protein extracted afterwards from it, in order to generate the binding site [19].

However, proteins have been a tricky material to carry out such tailoring processes, because they undergo conformational changes quite easily and have multiple charge locations varying with the specific conformation they exhibit. These critical points under the preparation of PIMmaybeavoidedbyusingmildconditions, preferably close to those in the native environment of the protein. This includes room temperature polymerization procedures and use of compatiblematerials. Inaddition, a way toimproveprotein binding to the synthetic material is to label the binding site with charged

monomers. This procedure was found successful on the preparation of PIM [18], but the effect of the charged labels on this binding site is yet to be proven.

The biosensor device integrating PIM for PSA detection should be coupled to simple and low cost procedures/apparatus, such as those of the potentiometric kind, one approach that has been proven successful [20]. Potentiometric sensors offer the advantage of selectivity, simplicity, being of good overall precision and accuracy [21]. The corresponding devices may be of very low cost when assemble using disposable syringe bodies and micropipette tips. This last approach has only been recently tested for an organic compound [22], and never been applied to monitor complex target analytes such as proteins.

Considering that the PIM material will be integrated in a biosensor device of electrical nature, it is reasonable to expect that it should be assembled on a compatible and low cost material support of good overall electrical performance. So the surface imprinting was made on graphene sheets, a 2D structure of special electrical features and low electrical noise [23]. Its large surface area is also expected to provide high rebinding capacity to the final PIM structure. Protein molecules will be located at the surface of the graphene sheets with high surface-to-volume ratio, thus generating an improved kinetics and accessibility to the generated binding sites and an extended template removal [24]. These features correlated to an improved accessibility of the target species to the binding site, as well as reduced binding times [25]. The use of graphene as support for protein imprint was only most recently report [26].

Thus, thepresentwork proposes a novelPIMfor PSA, supported by graphene and displaying charged labels on the binding site. N/PIM and NIMwereused as control materials tocheck theeffect of the charged labels upon the material performance. All the prepared materials were used as ionophores in membranes of conventional solid-contact carbon electrodes and the resulting biosensors evaluated in terms of binding features, calibration slopes, dynamic linear range, limit of detection, effect of $\mathrm{pH}$ and selectivity. The best membrane composition was used to prepare micropipette tipbased electrodes of very low detection limit and to analyse serum samples.

\section{Experimental}

\subsection{Reagents and solutions}

De-ionized water (conductivity $<0.054 \mu \mathrm{S} / \mathrm{cm}$ at $25{ }^{\circ} \mathrm{C}$ ) was employed. All chemicals were of analytical grade and used without further purification.

Graphite (nanopowder $<500 \mathrm{~nm}$ and mean pore size of $137 \mathrm{~A}^{\circ}$ ), potassium permanganate, sulphuric acid $95-97 \%$, hydrogen peroxide $30 \%$, hydrochloric acid $37 \%$, sodium chloride and sodium hydrogen carbonate were obtained from Merck. Human Prostate Specific Antigen (PSA), $N$-ethyl- $N$-(3-dimethylaminopropyl) carbodiimide hydrochloride (EDAC), 4-(2-hydroxyethyl)-1piperazineethanesulfonic acid (HEPES), trypsin, 2-Aminoethyl methacrylate hydrochloride 90\% (AMH), vinyl benzoate (VB), acrylamide (AA), N,N-methylenebis(acrylamide) (NMAA), creatinine, human hemoglobin, bovine serum albumin (BSA), urea and glucose were obtained from Sigma-Aldrich. Potassium nitrate, benzoyl peroxide (BOP) and tetrahydrofuran (THF) were obtained from Riedel-deHäen. o-Nitrophenyloctyl ether (oNPOE), poly(vinylchloride) (PVC) of high molecular weight, and $N$-hydroxysuccinimide (NHS) were obtained from Fluka, and (vinylbenzyl)trimethylammonium chloride $97 \%$ (VTA) was purchased to Acros Organics.

Stock solutions of PSA $2.5 \times 10^{4} \mathrm{ng} / \mathrm{mL}$ were prepared in Hepes $1 \times 10^{-4} \mathrm{~mol} / \mathrm{L}(\mathrm{pH} \sim 5.2)$ and less concentrated standard solutions were prepared by suitable dilution in the same buffer. The effect of $\mathrm{pH}$ was studied by changing the $\mathrm{pH}$ of a $50 \mathrm{~mL}$ PSA solution with a concentration of $7 \mathrm{ng} / \mathrm{mL}$. The $\mathrm{pH}$ alteration was achieved by little additions of either concentrated hydrochloric acid or saturated sodium hydroxide solution, freshly prepared. Selectivity studies used creatinine $(130 \mathrm{mg} / \mathrm{L})$, urea $(1900 \mathrm{mg} / \mathrm{L})$, glucose $(10.5 \mathrm{~g} / \mathrm{L})$, human hemoglobin $(150 \mathrm{~g} / \mathrm{L})$ and BSA $(50 \mathrm{~g} / \mathrm{L})$ solutions, prepared in Hepes buffer.

\subsection{Apparatus}

All potentiometric measurements were made in a Crison $\mathrm{pH}$ meter GLP $21( \pm 0.1 \mathrm{mV}$ sensitivity), at room temperature, and under constant stirring. The simultaneous reading of multiple potentiometric devices was enabled by a home-made commutation unit with six ways out. The assembly of the potentiometric cell using the solid-contact support was as follows: conductive graphite | PSA selective membrane | buffered solution (Hepes buffer $1 \times 10^{-4} \mathrm{~mol} / \mathrm{L}, \mathrm{pH} 5.2$, or artificial serum, $\mathrm{pH}$ 7.3) $\|$ electrolyte solution, $\mathrm{KCl}|\mathrm{AgCl}(\mathrm{s})| \mathrm{Ag}$. The reference electrode was an $\mathrm{Ag} / \mathrm{AgCl}$ electrode of double-junction from Crison, 5240.

The $\mathrm{pH}$ of solutions was measured by a Crison GLP 21 combined glass electrode connected to the above $\mathrm{pH}$ meter. An SBS vortex, MVOR03, was used to grant a good mixing of the reacting solutions. Insoluble materials were suspended in a Sonorex digitec sonicator. 
The chemical changes imposed to the materials were controlled by Fourier Transformed Infrared (FTIR) spectra, in Nicolet 6700 FTIR spectrometer coupled to an Attenuated Total Reflectance (ATR) sampling accessory of diamond contact crystal, also from Nicolet. Transmission electron microscopy (TEM) analysis was also conducted over the same materials, in a Hitachi H-9000 AT, operated at $200 \mathrm{kV}$. Raman spectroscopy studies were also conducted, using a LabRam 300 Jobin Yvon spectrometer, equipped with laser of $50 \mathrm{~mW}$ power operating at $532 \mathrm{~nm}$.

\subsection{Preparation of graphene oxide}

Graphene oxide (GO) was obtained from graphite powder by following the method of Hummers and Offeman [27], and its subsequent modifications described by Shenguang et al. [28]. Briefly, $2.0 \mathrm{~g}$ of graphite powder, $2.0 \mathrm{~g}$ of $\mathrm{KNO}_{3}$ and $6.0 \mathrm{~g}$ of $\mathrm{KMnO}_{4}$ were slowly added to $40 \mathrm{~mL}$ of concentrated $\mathrm{H}_{2} \mathrm{SO}_{4}$ under vigorous stirring at $0{ }^{\circ} \mathrm{C}$. The mixture was then stirred continuously for $1 \mathrm{~h}$ at ambient temperature. After that, $160 \mathrm{~mL}$ of water was added to the mixture and the temperature was increased up to $95^{\circ} \mathrm{C}$. The suspension was maintained at this temperature for $15 \mathrm{~min}$ and then poured into $240 \mathrm{~mL}$ of ultrapure water. After, $\sim 16 \mathrm{~mL}$ of $\mathrm{H}_{2} \mathrm{O}_{2}$ was added into thesuspension. Thesuspension wasimmediately cooled to room temperature, the solid products were filtered, washed with $5 \% \mathrm{HCl}$ aqueous solution and water, and dried. The obtained solid was finally dispersed in water to yield a yellow-brown suspension $(1 \mathrm{mg} / \mathrm{mL})$. This GO suspension was ultrasonicated for $10 \mathrm{~min}$ and then centrifuged for $5 \mathrm{~min}$ to remove the unexfoliated graphite oxide particles from it.

\subsection{Synthesis of protein imprinted material}

The overall scheme of synthesis may be found in Fig. 1 About $80 \mathrm{~mL}$ of $1 \mathrm{mg} / \mathrm{mL} \mathrm{GO}$ solution was mixed with $56 \mathrm{~mL}$ of a $50 \mathrm{mg} / \mathrm{mL}$ NHS aqueous solution. This solution was placed under continuous magnetic stirring and then added of $17.2 \mathrm{~mL}$ of fresh EDAC aqueous solution $(10 \mathrm{mg} / \mathrm{mL})$. This mixture was continuously stirred at room temperature for $30 \mathrm{~min}$. The colloidal GO so obtained started to flocculate and was removed from the solution by filtration, washed with a $1 \times 10^{-4} \mathrm{~mol} / \mathrm{L}$ Hepes buffer, and allowed to dry in a desiccator under nitrogen atmosphere.

For the preparation of PIM materials, about $1.5 \mathrm{mg}$ of the previous solid was immersed in $50 \mu \mathrm{L}$ of a $2.5 \times 10^{4} \mathrm{ng} / \mathrm{mL}$ PSA solution in Hepes buffer for protein binding. NIM materials were prepared in parallel by replacing the PSA solution by only Hepes buffer. Each resulting mixture was continually stirred at room temperature for $4 \mathrm{~h}$, and the solid was separated and washed with Hepes buffer $1 \times 10^{-4} \mathrm{~mol} / \mathrm{L}$. Then, $100 \mu \mathrm{L}$ of a $0.1 \mathrm{~g} / \mathrm{mL}$ AMH solution prepared in Hepes buffer was added to the solid. The obtained suspension was continually stirred at room temperature for $2 \mathrm{~h}$, and the solid washed with Hepes buffer $1 \times 10^{-4} \mathrm{~mol} / \mathrm{L}$.

The next stage consisted on the addition to the solid of $50 \mu \mathrm{L}$ of VTA solution, $2.9 \times 10^{-6} \mathrm{~g} / \mathrm{mL}, 50 \mu \mathrm{L}$ of $\mathrm{VB}$ solution, $6.8 \times 10^{-7} \mathrm{~g} / \mathrm{mL}$, for the preparation of plastic antibody material with charged binding sites (C/PIM or C/NIM), or $100 \mu \mathrm{L}$ of a $1.3 \times 10^{-6} \mathrm{~g} / \mathrm{mL}$ AA for the preparation of neutral materials (N/PIM orN/NIM). Bothsuspensionswerecontinually stirred atroom temperature for $2 \mathrm{~h}$.

The polymerization stage around the protein started by adding to the solid $100 \mu \mathrm{L}$ of a solution of $3.56 \times 10^{-4} \mathrm{~g} / \mathrm{mL}$ AA (functional monomer), $7.72 \times 10^{-3} \mathrm{~g} / \mathrm{mL}$ NMAA (cross-linker) and $1.2 \times 10^{-3} \mathrm{~g} / \mathrm{mL}$ BOP (radical initiator). The polymerization was carried out at room temperature for $2 \mathrm{~h}$. The resulting solids were washed again with Hepes buffer $1 \times 10^{-4} \mathrm{~mol} / \mathrm{L}$. Finally, $50 \mu \mathrm{L}$ of a $0.5 \mathrm{~g} /$ Ltrypsinsolutionwasadded tothesolid, and theresultingsuspension kept under continuous stirring, at room temperature, for 2h. The obtained materials (C/PIM, C/NIM, N/PIM and N/NIM) were centrifuged, washed with Hepes buffer and dried in a desiccator under nitrogen atmosphere.

\subsection{Assembly of the potentiometric sensors}

The selective membranes were prepared by mixing $1 \mathrm{mg}$ of modified graphene material (C/PIM, C/NIM, N/PIM or N/NIM), $33 \mathrm{mg}$ of oNPOE, and $16 \mathrm{mg}$ of PVC (Table 1 ). The mixture was stirred until the PVC was well humidified, and dispersed in $2.0 \mathrm{~mL}$ THF. The dispersion was kept uniform by continuous agitation on a magnetic stirrer.

The construction of the solid-contact PSA selective electrode was made similarly to that described by Kamel et al. in [29]. The electrode body was replaced by using a $10 \mathrm{~mL}$ syringe, using the smaller end to pack the conductive material (a mixture of graphite and epoxy resin) and bind the cupper electrical wire. The outer graphite layer on top of the syringe was removed to create a small

cavity ( $\sim 1 \mathrm{~mm}$ deep), where the selective membrane would be deposited, drop-by-drop. After application of the membrane solution, the membrane was allowed to dry for 24 hours and after conditioned in a solution PSA, $20 \mathrm{ng} / \mathrm{mL}$ in Hepes buffer. Due to the instability of PSA, this conditioning was made inside the fridge.

The electrodes of internal reference solution were constructed by following the procedures described by Almeida et al., in [22]. Only the best membrane composition was applied into the electrode bodies made from $1000 \mu \mathrm{L}$ micropipette tips made of polypropylene. The membrane solution was applied by dipping the tip about $4 \mathrm{~mm}$ inside the membrane solution. The membrane that entered the tip was allowed to dry for 24 hours. A silver wire covered with a thin layer of $\mathrm{AgCl}$ was introduced inside the micropipette body to serve as electrical connection to the inner reference solution. The composition of the inner reference solution was identified after the optimization procedures described later.

\subsubsection{Procedures for potentiometric measurements}

All potentiometric measurements were carried out at room temperature and in stirred solutions. Emf values of each electrode were measured in solutions with fixed $\mathrm{pH}$ 7.3.

Decreasing concentration levels of PSA were obtained by transferring $5 \mu \mathrm{L}$ of PSA aliquots of PSA $2.5 \times 10^{4}$ or $2.5 \times 10^{3} \mathrm{ng} / \mathrm{mL}$ standard solution to a $75 \mathrm{~mL}$ beaker containing $50 \mu \mathrm{L}$ of artificial serum and $950 \mu \mathrm{L}$ buffer $1.0 \times 10^{-4} \mathrm{~mol} / \mathrm{L}$. Potential readings were recorded after stabilization to $\pm 0.2 \mathrm{mV}$ and emf was plotted as a function of logarithm PSA concentration. Each calibration plot was used for subsequent determination of unknown PSA concentrations. The concentration interval of the calibration was $2.0-124.4 \mathrm{ng} / \mathrm{mL}$ or $0.2-12.4 \mathrm{ng} / \mathrm{mL}$ for electrodes made with solid-contact or inner reference solution, respectively. The artificial serum with different concentrations of PSA for the evaluation of ISE response was obtained by adding a known amount of PSA ( 2.5 to $60 \mathrm{ng} / \mathrm{mL}$ ) to artificial serum.

\subsubsection{Binding experiments}

Binding constants were calculated by theSandwich method. For this purpose, the conductive support of the ISE was first coated with NIM membranes, left to dry for 1 hour, and then coated with PIM membranes and let to dry for 24 hours. Before use, the sensors were let stand for 12 hours in a solution PSA, $20 \mathrm{ng} / \mathrm{mL}$ in Hepes buffer, in the fridge. The sensing head of the ISE were then submerged in a solution of $14 \mu \mathrm{L}$ of PSA $\left(2.5 \times 10^{4} \mathrm{ng} / \mathrm{mL}\right), 2.5 \mathrm{~mL}$ deserumartificial and $47.5 \mathrm{~mL}$ of buffer $1.0 \times 10^{-4} \mathrm{~mol} / \mathrm{L}$. The emf was then recorded until full stabilization. 


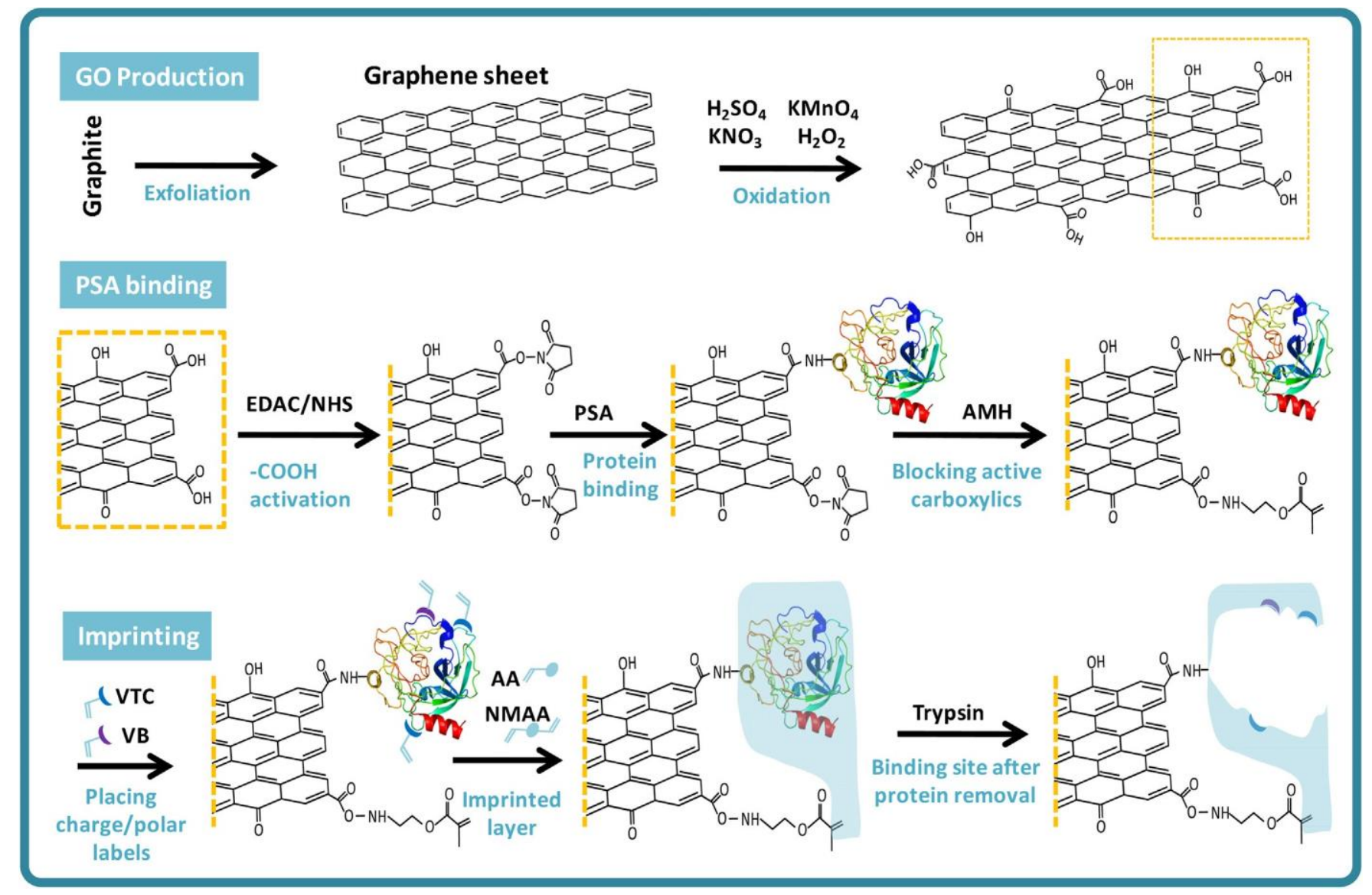

Fig. 1. Scheme for synthesis of the C/PIM materials (N/PIM, C/NIM, N/NIM are obtained by omitting specific steps of this scheme).

\subsubsection{Surface analysis (FTIR, TEM and Raman)}

The chemical alteration of the graphene was followed by FTIR analysis. The infrared spectra were collected after background correction. Each spectrum was the average of 32 scans for the same sample. The plot represented wave number, with a range from 600 to $4,000 \mathrm{~cm}^{-1}$, in function of \% transmittance. Resolution was 1:4000.

The TEM analysis was performed for PIM, NIM and oxidized graphene. All these materials were dry, dispersed in ethanol and deposited on a copper grid with a perforated film prior to microscopic observation. The analysis was done at several sampling points for each material.

Raman spectra were recorded as an extended scan; the laser beam was focused either with $50 \times$ or $100 \times$ Olympus objective lens and the laser power at the surface of the samples was varied with the aid of a set of neutral density filters (optical densities 0.3, 0.6, 1 and 2).

\section{Results and discussion}

\subsection{Plastic antibody design}

The overall design of the plastic antibody is presented in Fig. 1. Graphene was the physical support selected to carry out the imprinting process. It consists in a two-dimensional monolayer of carbon atoms where most of all have $\mathrm{sp}^{2}$ hybridization, offering a unique environment for fast electron transport [30]. Graphene was obtained by exfoliating graphite material, a process that ended up with the formation of GO. GO contains several functional groups, including hydroxyl $(-\mathrm{OH})$, carbonyl $(-\mathrm{C}=\mathrm{O})$, and carboxyl $(-\mathrm{COOH})$ [31].

The next stage was to bind PSA to the GO material to enable its subsequent imprint (Fig. 1). For this purpose, it was necessary to activate the carboxylic functions within the GO lattice, thus allowing the subsequent binding under mild conditions of any amine

Table 1

Membrane composition of PSA sensors casted in $15.6 \mathrm{mg}$ of PVC and their potentiometric features in $1.0 \times 10^{-4} \mathrm{~mol} / \mathrm{L}$ Hepes buffer.

\begin{tabular}{|c|c|c|c|c|c|c|c|c|c|c|}
\hline \multirow[t]{2}{*}{ ISE } & \multicolumn{3}{|c|}{ Membrane composition } & \multirow[t]{2}{*}{ Slope (mV/decade) } & \multicolumn{2}{|l|}{ LOD } & \multirow[t]{2}{*}{$R^{2}(n=3)$} & \multicolumn{2}{|c|}{ Linear concentration range } & \multirow[t]{2}{*}{$\mathrm{a}_{\mathrm{v}}(\mathrm{mV})$} \\
\hline & Ionophore (mg) & oNFOE (mg) & PVC (mg) & & (ng/mL) & $(\mathrm{mol} / \mathrm{L})$ & & (ng/mL) & $(\mathrm{mol} / \mathrm{L})$ & \\
\hline I & 0.9 (C/PIM) & 38.9 & 15.6 & $-44.16 \pm 2.89$ & $<2.0$ & $<5.83 \times 10^{-11}$ & 0.991 & $2.0-89.0$ & $5.83 \times 10^{-11}-2.62 \times 10^{-9}$ & 0.67 \\
\hline II & 0.95 (C/NIM) & 30.4 & 15.6 & $-24.80 \pm 1.91$ & 2.0 & $5.83 \times 10^{-11}$ & 0.994 & $2.6-89.0$ & $7.64 \times 10^{-11}-2.62 \times 10^{-9}$ & 0.90 \\
\hline III & 0.9 (N/PIM) & 35.8 & 15.6 & $-35.82 \pm 1.21$ & 2.0 & $5.83 \times 10^{-11}$ & 0.992 & $2.6-59.4$ & $7.64 \times 10^{-11}-1.75 \times 10^{-9}$ & 1.10 \\
\hline IV & $0.85(\mathrm{~N} / \mathrm{NIM})$ & 34.8 & 15.5 & $-28.25 \pm 1.4$ & 3.8 & $1.11 \times 10^{-10}$ & 0.996 & $3.8-40.3$ & $1.11 \times 10^{-10}-1.18 \times 10^{-9}$ & 1.15 \\
\hline $\mathrm{V}$ & - & 34.0 & 15.5 & - & - & - & - & - & - & - \\
\hline
\end{tabular}

LOD: Limit of detection 
group in outer surface of the protein (hard conditions would promote significant changes in the protein conformation, thus leading to a mismatch imprint). This activation was done by the conventional biochemical reaction involving EDAC/NHS transformation [32]. The reaction with PSA was made next and resulted in the formation of an amide bond that prevented the protein from moving out from the solid support. The carboxylic groups that remained active after the reaction with PSA were blocked by reaction with $\mathrm{AMH}$ (Fig. 1). AMH combined in the same structure a vinyl group and an amine function: the amine reacted with the activated carboxylic groups and blocked their reactivity, while the vinyl group was expected to participate in the subsequent polymeric reaction leading to imprint, thus ensuring that the imprinted polymer formed around the protein would be covalently attached to the graphene support.

The imprinting stage started by introducing charge/polar labels (C) in the binding site of the imprinted material (C/PIM). This was done by adding to the solution charged/polar monomeric structures: VTA with a positive quaternary ammonium salt and VB with an ester function providing a negative polarity. Both of these contained (as $\mathrm{AMH}$ ) a vinyl group that would enable their subsequent binding to the imprinted polymeric network. The molar amount of these monomers was controlled to avoid their binding out from the protein surface. VTA was also present in a higher molar amount due to the negative overall net charge of PSA under physiological conditions.

The imprinting around the protein with the charged labels was made by polymerizing AA cross linked by NMAA. The polymerization was initiated by BOP radicals. The imprinted sites were obtained by removing the protein template with trypsin, a protease that digests proteins by destroying peptide bonds. Negative controls of the above process were made by imprinting without template (C/NIM or N/NIN) and withoutcharged labels (N/PIM and N/NIM).

\subsection{Control of graphene modification}

The chemical modification made on GO to establish the protein imprinting was followed by different techniques, namely FTIR, Raman and TEM analysis. The results obtained for C/PIM, N/PIM, $\mathrm{C} / \mathrm{NIM}$ and N/NIM materials are shown in Fig. 2, and compared to GO as starting material.

The FTIR spectrum of GO (Fig. 2, top-left) presented a strong absorption peak at $\sim 1700 \mathrm{~cm}^{-1}$, that evidences the presence of the carbonyl group $(-\mathrm{C}=\mathrm{O})$. The broad adsorption band between 3700 and $3000 \mathrm{~cm}^{-1}$ indicated the presence of carboxylic function $(-\mathrm{COOH})$, as well as the unsaturation between carbon atoms with double bonds and the subsequent $\mathrm{sp}^{2}$ hybridization of these carbon atoms. The peaks at 1210 and $1070 \mathrm{~cm}^{-1}$ are probably accounting the presence of hydroxyl groups $(-\mathrm{OH})$ in the $\mathrm{GO}$ due to $\mathrm{C}-\mathrm{O}$ stretching vibrations. All materials obtained after GO modification showed similar FTIR spectra, with major depreciation of the significant absorption bands/regions observed in GO. This change in FTIR spectra accounted the presence of the polymeric network around graphene sheets. This similar behaviour was already expected because these materials differed only in the special arrangement of the polymeric network and had roughly the same chemical composition. The only chemical difference within these is that " $\mathrm{C}$ " materials include charged monomers, but these are present in very low amount becoming imperceptible under FTIR studies.

The Raman spectra of all materials were dominated by two bands (Fig. 2, bottom-left). These are the so-called G band, typically associated to the in-phase vibration of the graphite lattice, and the $\mathrm{D}$ band, corresponding to the (weak) disorder band from graphite edges [33]. The absolute intensities of $G$ and D peaks in C/PIM, N/PIM, C/NIM and N/NIM were much higher than those in GO and quite similar within this group of materials. This common observation among the modified graphene-based materials resulted from the similar modification made on GO: the presence of the polymeric layer on the graphene sheets. In addition to this increase in peak intensity, the chemical modification of GO changed the intensity ratio D/G band which reflects the extent of disorder present within the material [34]. The 1.03 ratio observed in GO changed to 0.96 in C/PIM and C/NIM and to 0.99 in N/PIM and N/PIM materials. This change was thus correlated to the polymeric material present in the graphene sheets, also reflecting the presence of charged monomers within the polymer matrix.

The TEM images obtained were not as helpful as Raman in terms of chemical modification of the GO. Graphene-sheets are not "hard" and are very thin, for which they were captured in electronmicroscope images in many different positions. Only theimprinted versions of the material showed small black dots coupled to the sheets (Fig. 2, right), meaning that these dots may be correlated to the binding sites. Such correlation is however difficult to confirm because the observed material is highly heterogeneous (GO or the other derived materials).

\subsection{Performance of the Sensors}

PSA sensors were prepared with PIM or NIM particles, with or without charged labels, acting as electroactive materials. These materials were dispersed in plasticized PVC and casted over a solid conductive contact made of graphite and epoxy resin. The main analytical features of the devices were obtained by calibrating the electrochemical cell in a range of concentrations of PSA between $5.83 \times 10^{-11}$ and $2.62 \times 10^{-9} \mathrm{~mol} / \mathrm{L}(2.0$ and $89.0 \mathrm{ng} / \mathrm{mL})$ under static mode of operation. The analytical data extracted from these were calculated according to IUPAC recommendations [35].

The obtained results are presented in Table 1. Overall, C/PIM sensor showed the best potentiometric response, with slopes of $44.2 \mathrm{mV} /$ decade and LOD below $5.83 \times 10^{-11} \mathrm{~mol} / \mathrm{L}$, in agreement with the results depicted in Fig. 3A. Furthermore, the nonimprinted versions showed smaller sensitivity and showed liner responses for higher concentrations, meaning that the imprinting stage was important to promote a more directed response for PSA. The charged labels were also important, increasing the sensitivity of the response and the reducibility of the obtained signals, both in imprinted and non-imprinted materials. This result may also account to the increase in perm-selectivity obtained by the presence of charged sites inside the selective membrane, besides suggesting that the labels increased the ability of the material to bind PSA. In general, the time required for the electrodes to reach a steady potential $( \pm 0.2 \mathrm{mV})$ was less than $20 \mathrm{~s}$, even for the highest tested concentrations. The response of the electrodes was fully reversible, a common feature among most potentiometric membranes selective electrodes. The same electrode could be recalibrated several times along one day and several consecutive days, within 2 months (Fig. 4). In addition, no significant potential changes in absolute values have been observed over this period. The response was also reproducible along this time, as reflected by the av presented in Table1.

The above results were supported by further binding studies, carried out by the sandwich method. For this purpose, membranes of C/PIM and N/PIM materials were casted on top of the corresponding blank membranes, i.e. C/NIM and N/NIM, respectively. The average binding constants so obtained for C/PIM and N/PIM materials were 2.67 and 1.55, respectively, showing the importance of the charged sites within the imprinted layer. These results also suggest that a substantial part of the potentiometric response may arise from a stereochemical recognition of the analyte at the imprinted sites. 


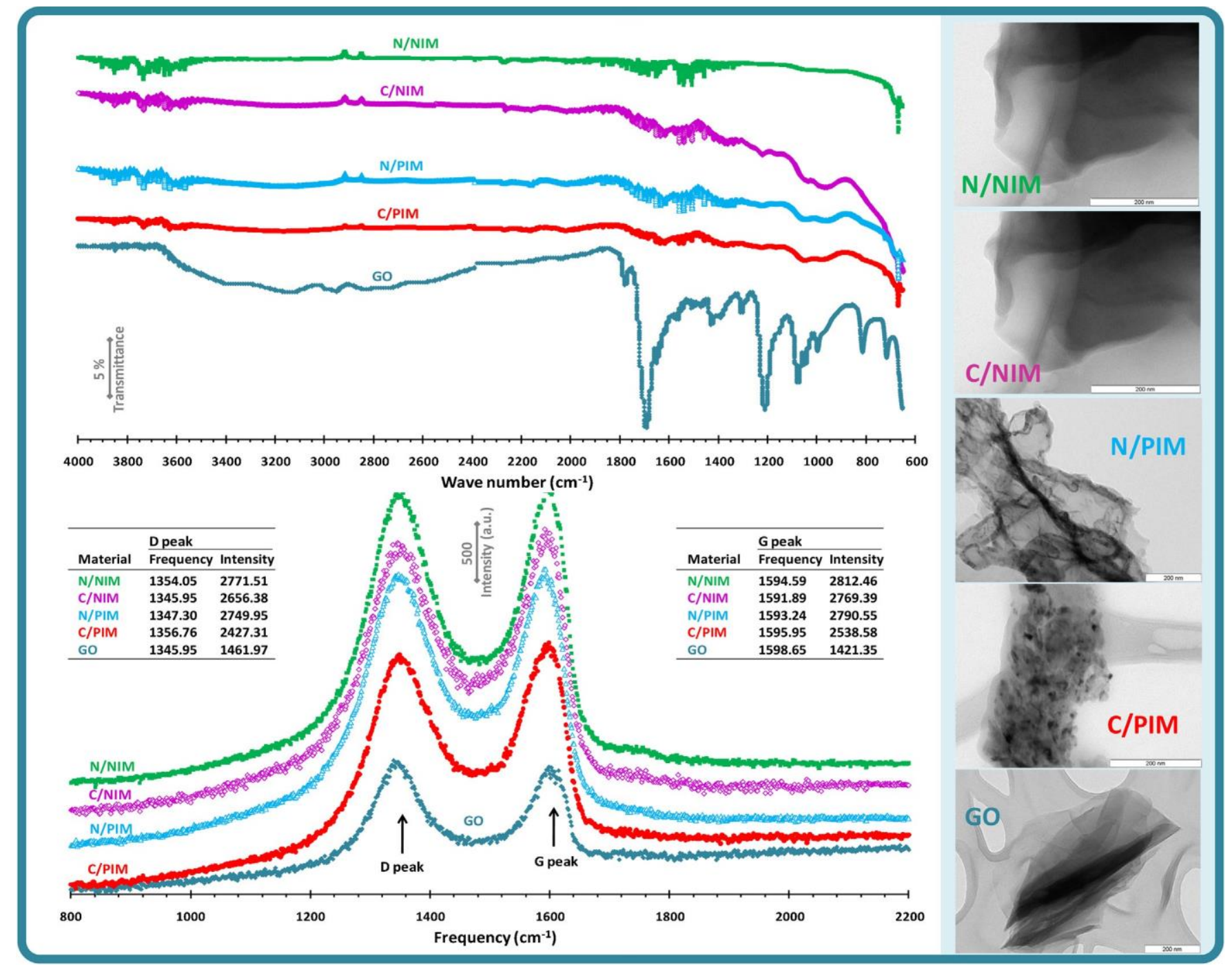

Fig. 2. FTIR (top, left) and Raman (bottom, left) spectra and TEM images (right) of all materials (GO is presented as blank control).

\subsection{Effect of $p H$}

The $\mathrm{pH}$ is an important variable for an accurate PSA reading, mostly because potentiometric sensors detect charged species and PSA is a multiple charged structure, with a net charge that depends from $\mathrm{pH}$ of the reading solution comparing to its isoelectric point [36].

The $\mathrm{pH}$ effect on the potentiometric devices was studied by recording Reilley diagrams, plotting the emf variation of a solution of constantPSA concentration with varying $\mathrm{pH}$ values. The concentration of PSA in thisstudywassetto $7 \mathrm{ng} / \mathrm{mL}$, and thepHwasvaried from 12 and 2, by adding saturated $\mathrm{NaOH}$ solution (to set the $\mathrm{pH}$ to 12) and small aliquots of concentrated $\mathrm{HCl}$ solutions (to decrease slightly the $\mathrm{pH}$, until pH2). Both C/PIMand N/NIMshowed a similar behaviour: emfs varied less than $20 \mathrm{mV}$ within the $\mathrm{pH}$ interval 4-11; the emf increased below $\mathrm{pH} 4$, accounting for the intense positive charge in PSA and a possible $\mathrm{H}^{+}$interference; and the emf decreased below $\mathrm{pH} 11$, in result of the deprotonation of PSA and its negative netcharge.

According to the obtained results, and considering the wide $\mathrm{pH}$ range achieved with the above devices, the $\mathrm{pH}$ selected for subsequent studies was 7.2. This value is expected to be close or similar to physiological conditions, meaning that any future analytical application would have little or no requirements of $\mathrm{pH}$ adjustment.

\subsection{Sensor selectivity}

The selectivity behaviour of potentiometric sensors is typically expressed in potentiometric selectivity coefficients $\left(\mathrm{K}^{\mathrm{POT}}\right)$ [37], and lower values of $\mathrm{K}^{\mathrm{POT}}$ mean lower interference. The selectivity coefficients were assessed in this work by the matched potential method (MPM) [38], where the PSA concentration was changed from 4 to $10 \mathrm{ng} / \mathrm{mL}$ (leading to a $13 \mathrm{mV}$ emf change). The effect of foreign specifies upon a $4 \mathrm{ng} / \mathrm{mL}$ solution of PSA was checked for creatinine, urea, glucose, haemoglobin (human) and bovine serum albumin (BSA). These foreign species were included in this study because they are commonly present in human serum and may interfere in readings of PSA in serum samples.

Overall, the addition of small aliquots of solutions of foreign species was unable to change the emf in $13 \mathrm{mV}$, as requested to calculate the $\mathrm{K}^{\mathrm{POT}}$. This was tried out for highly concentrated solutions of interfering species and for concentrations up to their physiological levels. Facing this limitation, instead of calculating the potentiometric selectivity coefficient, tolerance levels were calculated for each foreign species. The concentrations of creatinine, urea, glucose, hemoglobin and BSA tolerated by the devices were $1.3 \times 10^{5}, 1.9 \times 10^{6}, 1.1 \times 10^{7}, 1.5 \times 10^{8}$ and $5.0 \times 10^{7} \mathrm{ng} / \mathrm{mL}$. In general, negligibleinterference was found for the foreign species under study tested up to the previously tolerated concentrations (higher 

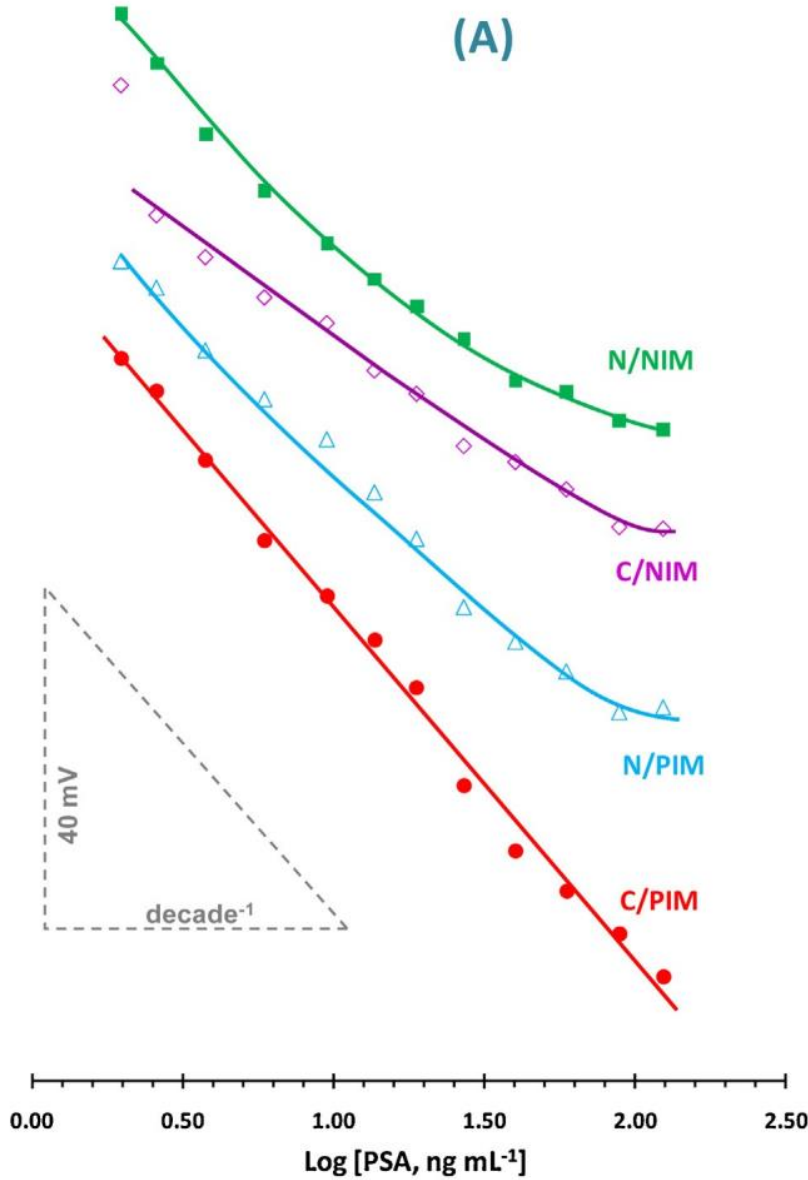

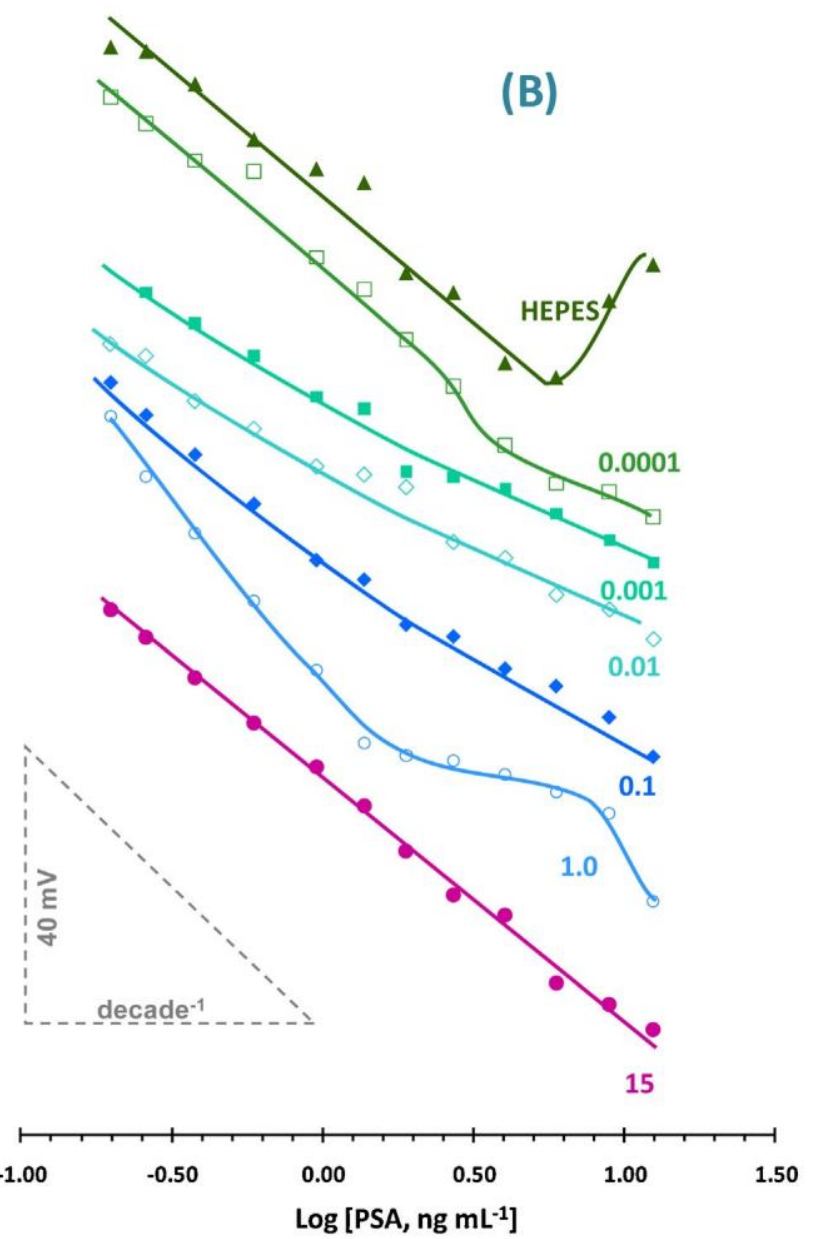

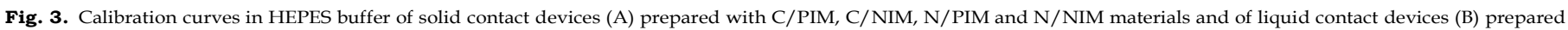
with C/PIM material and inner reference solutions of different PSA concentrations (expressed in nmol/L)

concentrations were not tested due to technical limitations in increasing the foreign species concentration withoutsignificantly changing the background concentration in PSA, set to $4 \mathrm{ng} / \mathrm{mL}$ due to its high clinical significance).

\subsection{Liquid contact ISEs}

Further optimization of the proposed sensor was tried out by applying the selected C/PIM membrane over the smaller end of a $1000 \mu \mathrm{L}$ micropipette tip and varying the inner reference solution composition. The concentration of the primary ion in the inner compartment is expected to be set to a low value, in order to generate a net flux of primary ions towards this side of the membrane. The exact concentration required for this purpose must be set by experimental studies.

Thus, several electrodes with different PSA concentrations in the inner electrolyte were constructed. The inner electrolyte was always an HEPES $1 \times 10^{-4} \mathrm{~mol} / \mathrm{L}$ buffer, with PSA concentrations

Table 2

Comparison of PSA sensors with different inner electrolyte solutions.

\begin{tabular}{|c|c|c|c|c|c|c|c|}
\hline \multirow[t]{2}{*}{ Characteristics } & \multicolumn{4}{|l|}{ PSA (mol/L) } & \multicolumn{3}{|l|}{ Hepes Buffer } \\
\hline & $\begin{array}{l}1.53 \times 10^{-8} \\
I\end{array}$ & $\begin{array}{l}1 \times 10^{-9} \\
I I\end{array}$ & $\begin{array}{l}1 \times 10^{-10} \\
I I I\end{array}$ & $\begin{array}{l}1 \times 10^{-11} \\
I V\end{array}$ & $\begin{array}{l}1 \times 10^{-12} \\
V\end{array}$ & $\begin{array}{l}1 \times 10^{-13} \\
V I\end{array}$ & $\begin{array}{l}- \\
V I I\end{array}$ \\
\hline Slope (mV/decade) & $-34.04 \pm 1.24$ & $-50.64 \pm 0.38$ & $-31.19 \pm 3.57$ & $-23.52 \pm 1.30$ & $-23.77 \pm 0.85$ & $-35.46 \pm 6.04$ & $-34.57 \pm 2.75$ \\
\hline$R^{2}(n=3)$ & 0.995 & 0.991 & 0.990 & 0.991 & 0.992 & 0.992 & 0.992 \\
\hline LOD (ng/mL) & 0.2 & 0.2 & 0.2 & 0.2 & 0.2 & 0.2 & 0.2 \\
\hline $\mathrm{LOD}(\mathrm{mol} / \mathrm{L})$ & $5.83 \times 10^{-12}$ & $5.83 \times 10^{-12}$ & $5.83 \times 10^{-12}$ & $5.83 \times 10^{-12}$ & $5.83 \times 10^{-12}$ & $5.83 \times 10^{-12}$ & $5.83 \times 10^{-12}$ \\
\hline LLLR (ng/mL) & 0.2 & 0.2 & 0.2 & 0.2 & 0.2 & 0.2 & 0.2 \\
\hline $\operatorname{LLLR}(\mathrm{mol} / \mathrm{L})$ & $5.83 \times 10^{-12}$ & $5.83 \times 10^{-12}$ & $5.83 \times 10^{-12}$ & $5.83 \times 10^{-12}$ & $5.83 \times 10^{-12}$ & $5.83 \times 10^{-12}$ & $5.83 \times 10^{-12}$ \\
\hline ULLR (ng/mL) & 12.5 & 1.9 & 2.7 & 12.5 & 12.5 & 6.0 & 6.0 \\
\hline ULLR (mol/L) & $3.67 \times 10^{-10}$ & $5.57 \times 10^{-11}$ & $7.99 \times 10^{-11}$ & $3.67 \times 10^{-10}$ & $3.67 \times 10^{-10}$ & $1.75 \times 10^{-10}$ & $1.75 \times 10^{-10}$ \\
\hline$a_{V}(m V)$ & 0.64 & 0.035 & 0.093 & 0.98 & 0.75 & 1.56 & 1.92 \\
\hline
\end{tabular}

LLLR-Lower limit of linear range; ULLR-Upper limit of linear range 


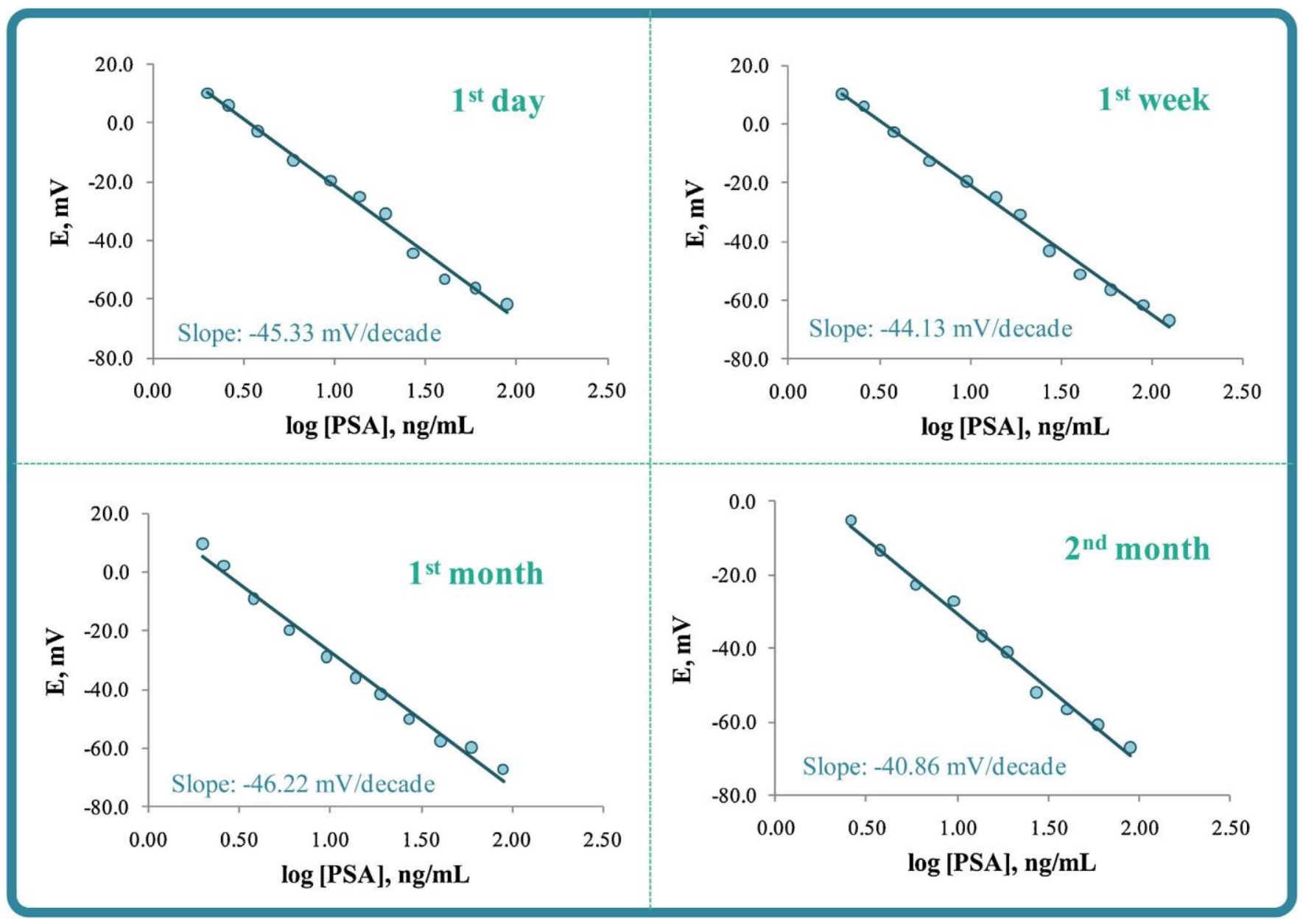

Fig. 4. Several calibrations of the C/PIM device measured with the same electrode, under equal background conditions and within time.

ranging from $1 \times 10^{-13}$ to $1.53 \times 10^{-8} \mathrm{~mol} / \mathrm{L}$, or without PSA. The obtained results are presented in Table 2 and the corresponding calibrations presented in Fig. 3B. Overall, the main differences recorded for all conditions tested were slope and linear range, being the best results obtained for the higher PSA concentration tested (which was already sufficiently low for this kind of electrodes). The LOD decrease 10 times, comparing to the solid contact electrodes, meaning that this kind of configuration may be especially attractive for screening vestigial amounts of protein biomarkers.

\subsection{Application}

C/PIM sensors were used to determine PSA in artificial serum. Blank serum samples were spiked and analyzed for PSA concentrations ranging about 2.6 to $59.4 \mathrm{ng} / \mathrm{mL}$.

The results of the potentiometric analysis conducted insteady state are summarized in Table 3. A good agreement was found

Table 3

Potentiometric determination of PSA in serum using MIP oriented based membrane sensor.

\begin{tabular}{lllll}
\hline PSA $(\mathrm{ng} / \mathrm{mL})$ & Found $(\mathrm{ng} / \mathrm{mL})$ & Recovery $(\%)$ & Relative error $(\%)$ & RSD $(\%)$ \\
\hline 59.4 & $52.0 \pm 5.6$ & $96.9 \pm 2.6$ & 3.1 & 10.7 \\
18.9 & $18.0 \pm 0.1$ & $98.1 \pm 0.3$ & 1.9 & 0.8 \\
9.5 & $9.1 \pm 1.1$ & $97.7 \pm 5.3$ & 2.3 & 11.9 \\
5.9 & $7.3 \pm 1.2$ & $111.9 \pm 9.0$ & -11.9 & 15.9 \\
3.8 & $4.0 \pm 0.3$ & $103.9 \pm 5.4$ & -3.9 & 7.2 \\
2.6 & $2.7 \pm 0.5$ & $106.1 \pm 18.1$ & -6.1 & 17.0 \\
\hline
\end{tabular}

between added and found amounts of PSA. Overall, recoveries ranged from 96.9 to $106.1 \%$ with an average relative standard deviation of $6.8 \%$, suggesting that the proposed sensors may have successful results under real applications.

\section{Conclusions}

The technique of molecular imprinting over graphene layers produced an inexpensive material that was successfully applied to produce PSA sensors of potentiometric transduction, being the presence of charged labels beneficial for the production of a more sensitive response, extensive to lower PSA concentrations. The use of a liquid contact allows a decrease in delectability, although the solid contact devices are more easy to use in routine applications are capable of reading directly PSA concentrations with clinical significance in serum.

The main advantages of these sensors include the simplicity of construction, low detection limits and low manufacturing costs. When compared to methods relying on natural antibodies, the present devices also offer reusability over 2 months. The proposed method is particularly suitable for screening assays carried out in analytical laboratories.

\section{Acknowledgement}

This work was supported by FCT, Foundation for Science and Technology through the PhD grant ref. SFRH/BD/79221/2011. 


\section{References}

[1] W.H.O. (WHO), http://www.who.int/mediacentre/factsheets/fs297/en/ index.html,2008

[2] S. Michel, G. Deléage, J.-P. Charrier, J. Passagot, N. Battail-Poirot, G. Sibai, M. Jolivet, C. Jolivet-Reynaud, Anti-Free Prostate-specific Antigen Monoclonal Antibody Epitopes Defined by Mimotopes and Molecular Modeling, Clinical Chemistry 45 (1999) 638-650.

[3] Y. Liu, Electrochemical detection of pro state-specific antigen based on gold colloids/alumina derived sol-gel film, Thin Solid Films 516 (2008) 1803-1808.

[4] H. Karsten, M. Klaus, Molecularly Imprinted Polymers and Their Use in Biomimetic Sensors, Chemical reviews 100 (2000) 2495-2504.

[5] J.F. Rusling, G. Sotzing, F. Papadimitrakopoulosa, Designing nanomaterialenhanced electrochemical immunosensors for cancer biomarker proteins, Bioelectrochemistry 76 (2009) 189-194.

[6] J. Szucs, E. Pretsch, R.E. Gyurcsanyi, Potentiometric enzyme immunoassay using miniaturized anion-selective electrodes for detection, Analyst 134 (2009) 1601-1607.

[7] W. Xiu-Ling, T. Guan-Hong, M. Yuan-Hug, Double-Layer Nanogold and Poly(amidoamine) Dendrimer-Functionalized PVC Membrane Electrode for Enhanced Electrochemical Immunoassay of Total Prostate Specific Antigen, Electroanalysis 21 (2009) 2109-2115.

[8] V. Escamilla-Gomez, D. Hernandez-Santos, M. Begona Gonzalez-Garcia, J. Manuel Pingarron-Carrazon, A. Costa-Garcia, Simultaneous detection of free and total prostate specific antigen on a screen-printed electrochemical dual sensor, Biosensors \& Bioelectronics 24 (2009) 2678-2683.

[9] C. Jang-Woo, K. Da-Yean, J. Yong-Hark, K. Hyun-Hee, M. Junhang, O. ByungKeun, Ultra-sensitive surface plasmon resonance based immunosensor for prostate-specific antigen using gold nanoparticle-antibody complex, Colloids and Surfaces a-Physicochemical and Engineering Aspects 313 (2008) 655-659.

[10] H. Jans, K. Jans, P.J. Demeyer, K. Knez, T. Stakenborg, G. Maes, L. Lagae, A simple double-bead sandwich assay for protein detection in serum using UV-vis spectroscopy, Talanta 83 (2011) 1580-1585.

[11] H.S.Jang, K.N.Park, C.D.Kang,J.P. Kim,S.J.Sim, K.S. Lee, Optical fiberSPRbiosensor with sandwich assay for the detection of prostate specific antigen, Optics Communications 282 (2009) 2827-2830.

[12] X. Wang, M. Zhao, D.D. Nolte, T.L. Ratliff, Prostate specific antigen detection in patient sera by fluorescence-free BioCD protein array, Biosensors \& Bioelectronics 26 (2011) 1871-1875.

[13] Z. Ye, M. Tan, G. Wang, J. Yuan, Preparation, characterization, and time-resolved fluorometric application of silica-coated terbium(ill) fluorescent nanoparticles, Analytical Chemistry 76 (2004) 513-518.

[14] K.W. Wee, G.Y. Kang, J. Park, J.Y. Kang, D.S. Yoon, J.H. Park, T.S. Kim, Novel electrical detection of label-free disease marker proteins using piezoresistive self-sensing micro-cantilevers, Biosensors \& Bioelectronics 20 (2005) 1932-1938.

[15] Y. Ding, H. Lu, G. Shi, J. Liu, G. Shen, R. Yu, Cell-based immobilization strategy for sensitive piezoelectric immunoassay of total prostate specific antigen, Biosensors \& Bioelectronics 24 (2008) 228-232.

[16] B. Zhang, X. Zhang, H.H. Yan, S.J. Xu, D.H. Tang, W.L. Fu, A novel multiarray immunoassay device for tumor markers based on insert-plug model of piezoelectric immunosensor, Biosensors \& Bioelectronics 23 (2007) 1925.

[17] Y. Uludag, I.E. Tothill, Development of a sensitive detection method of cancer biomarkers in human serum $(75 \%)$ using a quartz crystal microbalance sensor and nanoparticles amplification system, Talanta 82 (2010) 277-282.

[18] F.T.C. Moreira, S.Sharma, R.A.F. Dutra, J.P.C. Noronha, A.E.G.Cass, M.G.F.Sales, Smart Plastic Antibody Material (SPAM) tailored on disposable screen printed electrodes for protein recognition: application to Myoglobin detection, Biosensors and Bioelectronics 45 (2013) 237-244.
[19] F.T.C. Moreira, R.A.F. Dutra, J.P.C. Noronha, M.G.F. Sales, Surface Imprinting approach on screen printed electrodes coated with carboxylated PVC for Myoglobin detection with Electrochemical Transduction, Procedia Engineerin 47 (2012) 865-868

[20] F.T.C. Moreira, R.A.F. Dutra, J.P.C. Noronha, M.G.F. Sales, Myoglobin-biomimetic electroactive materials made by surface molecular imprinting on silica beads and their use as ionophores in polymeric membranes for potentiometric transduction, Biosens Bioelectron 26 (2011) 4760-4766.

[21] T.S.C.R. Rebelo, S.A.A. Almeida, J.R.L. Guerreiro, M.C.B.S.M. Montenegro, M.G.F. Sales, Trimethoprim-selective electrodes with molecularly imprinted polymers acting as ionophores and potentiometric transduction on graphite solidcontact, Microchemical Journal 98 (2011) 21-28.

[22] S.A.A. Almeida, L.A.A.N.A. Truta, R.B. Queiros, M.C.B.S.M. Montenegro, A.L. Cunha, M.G.F. Sales, Optimizing potentiometric ionophore and electrode design for environmental on-site control of antibiotic drugs: Application to sulfamethoxazole, Biosensors and Bioelectronics 35 (2012) 319-326.

[23] Y. Li, X. Li, C. Dong, J. Qi, X. Han, A graphene oxide-based molecularly imprinted polymer platform for detecting endocrine disrupting chemicals, Carbon 48 (2010) 3427-3433

[24] Y. Mao, Y. Bao, S. Gan, F. Li, L. Niu, Electrochemical sensor for dopamine based on a novel graphene-molecular imprinted polymers composite recognition element, Biosensors \& Bioelectronics 28 (2011) 291-297.

[25] T. Kuila, S. Bose, P. Khanra, A.K. Mishra, N.H. Kim, J.H. Lee, Recent advances in graphene-based biosensors, Biosensors and Bioelectronics 26 (2011) 46374648

[26] J. Luo, S. Jiang, X. Liu, Efficient One-PotSynthesis of Mussel-Inspired Molecularly Imprinted Polymer Coated Graphene for Protein-Specific Recognition and Fast Separation, The Journal of Physical Chemistry C 117 (2013) 18448-18456.

[27] W.S. Hummers, R.E. Offeman, Preparation of grafhitic oxide, Journal of the American Chemical Society 80 (1958) 1339.

[28] S. Ge, M. Yan, J. Lu, M. Zhang, F. Yu, J. Yu, X.Song, S. Yu, Electrochemical biosensor based on graphene oxide-Au nanoclusters composites for L-cysteine analysis, Biosensors \& Bioelectronics 31 (2012) 49-54.

[29] A.H. Kamel, F.T.C. Moreira, S.A.A. Almeida, M.G.F. Sales, Novel potentiometric sensors of molecular imprinted polymers for specific binding of chlormequat, Electroanalysis 20 (2008) 194-202.

[30] N.O. Weiss, H. Zhou, L. Liao, Y. Liu, S. Jiang, Y. Huang, X. Duan, Graphene An Emerging Electronic Material (Adv. Mater. 43/2012), Advanced Materials 24 (2012) 5776.

[31] V. Singh, D. Joung, L. Zhai, S. Das, S.I. Khondaker, S. Seal, Graphene based materials: Past, present and future, Progress in Materials Science 56 (2011) $1178-1271$.

[32] K. Jiang, L.S. Schadler, R.W. Siegel, X. Zhang, H. Zhang, M. Terrones, Protein immobilization on carbon nanotubes via a two-step process of diimideactivated amidation, Journal of Materials Chemistry 14 (2004) 37-39.

[33] F. Tuinstra, J.L. Koenig, Raman Spectrum of Graphite, Journal of Chemical Physics 53 (1970) 1126.

[34] K.N. Kudin, B. Ozbas, H.C. Schniepp, R.K. Prud'homme, I.A. Aksay, R. Car, Raman Spectra of Graphite Oxide and Functionalized Graphene Sheets, Nano Letters 8 (2007) 36-41.

[35] IUPAC, Analytical Chemistry Division Commission on Analytical Nomenclature, Pure and Applied Chemistry (2000) 1851-2082.

[36] L. Bruun, T. Bjork, H. Lilja, C. Becker, O. Gustafsson, A. Christensson, Percentfree prostate specific antigen is elevated in men on haemodialysis or peritoneal dialysis treatment, Nephrol Dial Transplant 18 (2003) 598-602.

[37] E. Bakker, E. Pretsch, Modern Potentiometry, Angewandte ChemieInternational Edition 46 (2007) 5660-5668.

[38] Y. Umezawa, P. Buhlmann, K. Umezawa, K. Tohda, S. Amemiya, Potentiometric selectivity coefficients of ion-selective electrodes Part I. Inorganic cations (Technical report), Pure and Applied Chemistry 72 (2000) 1851-2082. 